\title{
Microtubule and Actin Differentially Regulate Synaptic Vesicle Cycling to Maintain High-Frequency Neurotransmission
}

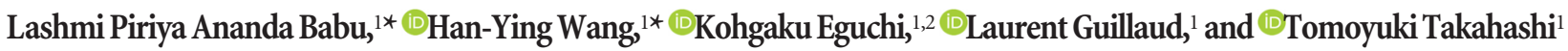 \\ ${ }^{1}$ Cellular and Molecular Synaptic Function Unit, Okinawa Institute of Science and Technology (OIST) Graduate University, Okinawa 904 - 0495, Japan, and \\ ${ }^{2}$ Molecular Neuroscience group, Institute of Science and Technology Austria (IST Austria), Am Campus 1, A-3400 Klosterneuburg, Austria
}

Cytoskeletal filaments such as microtubules (MTs) and filamentous actin (F-actin) dynamically support cell structure and functions. In central presynaptic terminals, F-actin is expressed along the release edge and reportedly plays diverse functional roles, but whether axonal MTs extend deep into terminals and play any physiological role remains controversial. At the calyx of Held in rats of either sex, confocal and high-resolution microscopy revealed that MTs enter deep into presynaptic terminal swellings and partially colocalize with a subset of synaptic vesicles (SVs). Electrophysiological analysis demonstrated that depolymerization of MTs specifically prolonged the slow-recovery time component of EPSCs from short-term depression induced by a train of highfrequency stimulation, whereas depolymerization of F-actin specifically prolonged the fast-recovery component. In simultaneous presynaptic and postsynaptic action potential recordings, depolymerization of MTs or F-actin significantly impaired the fidelity of high-frequency neurotransmission. We conclude that MTs and F-actin differentially contribute to slow and fast SV replenishment, thereby maintaining high-frequency neurotransmission.

Key words: F-actin; microtubules; neurotransmission; short-term depression; synaptic vesicles

Significance Statement

The presence and functional role of MTs in the presynaptic terminal are controversial. Here, we demonstrate that MTs are present near SVs in calyceal presynaptic terminals and that MT depolymerization specifically prolongs the slow-recovery component of EPSCs from short-term depression. In contrast, F-actin depolymerization specifically prolongs fast-recovery component. Depolymerization of MT or F-actin has no direct effect on SV exocytosis/endocytosis or basal transmission, but significantly impairs the fidelity of high-frequency transmission, suggesting that presynaptic cytoskeletal filaments play essential roles in SV replenishment for the maintenance of high-frequency neurotransmission.

\section{Introduction}

The cytoskeleton, comprising filamentous actin (F-actin), microtubules (MTs), and intermediate filaments, supports the cell ar-

\footnotetext{
Received July 3, 2019; revised Oct. 23, 2019; accepted Nov. 16, 2019.

Author contributions: L.P.A.B., L.G., and T.T. designed research; L.P.A.B., H.-Y.W., K.E., and L.G. performed research; L.P.A.B., H.-Y.W., K.E., and L.G. analyzed data; L.P.A.B., H.-Y.W., K.E., L.G., and T.T. wrote the paper.

This work was supported by the Okinawa Institute of Science and Technology. We thank Yasushi Okada for technical advice and access to the STED microscope. We also thank Tetsuya Hori, Yukihiro Nakamura, and Takeshi Sakaba for discussions and comments, and Steven D. Aird for editing this article.

*L.P.A.B. and H.-Y.W. contributed equally to this work.

The authors declare no competing financial interests.

Correspondence should be addressed to Laurent Guillaud at laurent.guillaud@oist.jp or Tomoyuki Takahashi at ttakahas@oist.jp.

https://doi.org/10.1523/JNEUROSCl.1571-19.2019

Copyright @ 2020 Piriya Ananda Babu, Wang et al.

This is an open-access article distributed under the terms of the Creative Commons Attribution License Creative Commons Attribution 4.0 International, which permits unrestricted use, distribution and reproduction in any medium provided that the original work is properly attributed.
}

chitecture and functions. During neuronal development, MTs and F-actin play crucial roles in cell motility, axonal growth, and organelle/protein transport to growth cones (Arnette et al., 2016). However, their functional roles in developed synapses remain unestablished. Among cytoskeletal elements, F-actin is localized at the distal end of presynaptic axons (Hirokawa et al., 1989; Saitoh et al., 2001). Functionally, it is thought to regulate neurotransmitter release (Morales et al., 2000), mediate endocytosis of synaptic vesicles (SVs; Watanabe et al., 2013; Delvendahl et al., 2016; Wu et al., 2016), and promote the recovery of synaptic responses from activity-dependent short-term depression (STD; Cole et al., 2000; Sakaba and Neher, 2003) via fast SV replenishment (Lipstein et al., 2013) and clearance of used SVs from release sites (Hosoi et al., 2009; Lee et al., 2012).

Compared with F-actin, much less is known about the presynaptic functional roles of MTs, except that they are involved in the 
transport of mitochondria and presynaptic elements such as those of active zones (AZs) or SVs (Hirokawa et al., 2010; Melkov and Abdu, 2018). Previous electron microscopy (EM) studies at the frog neuromuscular junction (NMJ) reported that MTs anchoring SVs are directing toward AZs (Gray 1978, 1983; Hirokawa et al., 1989). Likewise, at the Drosophila NMJ, the MTassociated protein Futsch (Hummel et al., 2000) links MT to AZs, thereby supporting neurotransmitter release (Lepicard et al., 2014). However, at lamprey or chick embryonic synapses, MTs do not colocalize with SVs (Smith et al., 1970; Bird, 1989). At the calyx of Held in adult cats, MTs are observed in presynaptic terminal swellings, but not in the SV pool (Perkins et al., 2010). In a recent imaging study in cultured calyceal presynaptic terminals, MTs are shown to be present within terminal swellings and depolymerization of MTs by nocodazole treatment impaired long-distance SV movements between swellings, whereas depolymerization of F-actin had no effect (Guillaud et al., 2017). However, in cultured hippocampal synapses, interbouton SV trafficking is reportedly blocked by an F-actin depolymerizing drug (Darcy et al., 2006).

To address whether MTs play any functional role in presynaptic terminals, we first examined their localization in the calyx of Held of rodent brainstem using confocal and stimulated emission depletion (STED) microscopy. We then depolymerized MTs in slices, using vinblastine, and examined whether it affects synaptic functions. For comparisons, we also depolymerized F-actin using latrunculin $\mathrm{A}$, and re-examined its effects on synaptic functional properties (Sakaba and Neher, 2003), newly at physiological temperature $\left(\mathrm{PT} ; 37^{\circ} \mathrm{C}\right)$ and at calyces of Held in posthearing rats. Our results revealed differential contributions of F-actin and MTs, specifically to fast and slow recovery of EPSCs from STD without cross talk, suggesting that these cytoskeletal elements independently contribute to recycling of SVs to maintain highfrequency neurotransmission at this fast synapse.

\section{Materials and Methods}

All experiments were performed in accordance with guidelines of the Physiological Society of Japan and animal experiment regulations at Okinawa Institute of Science and Technology Graduate University.

Slice preparation and solutions for electrophysiological recordings. Brainstems were isolated from Wistar rats of either sex, at the age of postnatal day 13-16, after decapitation under isoflurane anesthesia. Transverse brainstem slices (175-250 $\mu \mathrm{m}$ in thickness), containing the medial nucleus of the trapezoid body (MNTB), were cut using a vibroslicer (VT1200S, Leica) in ice-cold solution containing the following ( $\mathrm{mm})$ : 200 sucrose, $2.5 \mathrm{KCl}, 26 \mathrm{NaHCO}_{3}, 1.25 \mathrm{NaH}_{2} \mathrm{PO}_{4}, 6 \mathrm{MgCl}_{2}, 10$ glucose, 3 myo-inositol, 2 sodium pyruvate, and 0.5 sodium ascorbate, at $\mathrm{pH} 7.4$, when bubbled with $95 \% \mathrm{O}_{2}$ and $5 \% \mathrm{CO}_{2}$, and 310-320 mOsm. Before recordings, slices were incubated for $1 \mathrm{~h}$ at $37^{\circ} \mathrm{C}$ in artificial CSF (aCSF) containing the following (mM): $125 \mathrm{NaCl}, 2.5 \mathrm{KCl}, 1 \mathrm{MgCl}_{2}, 2 \mathrm{CaCl}_{2}, 10$ glucose, 3 myo-inositol, 2 sodium pyruvate, 0.5 sodium ascorbate, 1.25 $\mathrm{NaH}_{2} \mathrm{PO}_{4}$, and $26 \mathrm{NaHCO}_{3}$, at pH 7.4, when bubbled with $95 \% \mathrm{O}_{2}$ and $5 \% \mathrm{CO}_{2}$, and 310-315 mOsm. For recording EPSCs, the aCSF contained $10 \mu \mathrm{M}$ bicuculline methiodide (Sigma-Aldrich) and $0.5 \mu \mathrm{M}$ strychnine hydrochloride (TCI) to block $\mathrm{GABA}_{\mathrm{A}}$ and glycine receptors, respectively. The pipette solution for recording postsynaptic currents contained the following (mM): $110 \mathrm{CsF}, 30 \mathrm{CsCl}, 10$ HEPES, 5 EGTA, $1 \mathrm{MgCl}_{2}$, and 5 QX314-Cl, at pH 7.3-7.4, adjusted with $\mathrm{CsOH}$, and 300-320 mOsm, unless otherwise noted. For presynaptic membrane capacitance measurements, the aCSF contained $10 \mathrm{~mm}$ tetraethylammonium chloride (TCI), $0.5 \mathrm{~mm} 4$-aminopyridine (Nacalai Tesque), $1 \mu \mathrm{m}$ tetrodotoxin (Nacalai Tesque), $10 \mu \mathrm{m}$ bicuculline methiodide, and $0.5 \mu \mathrm{m}$ strychnine hydrochloride. The pipette solution for presynaptic membrane capacitance $\left(C_{\mathrm{m}}\right)$ measurements contained the following $(\mathrm{mm})$ : $105 \mathrm{Cs}$ gluconate, $30 \mathrm{CsCl}$, 10 HEPES, 0.5 EGTA, 12 disodium phosphocreatinine,
$3 \mathrm{Mg}$-ATP, $0.3 \mathrm{Na}_{2}$-GTP, and $1 \mathrm{MgCl}_{2}$, at $\mathrm{pH}$ 7.3-7.4, adjusted with $\mathrm{CsOH}$, and 315-320 mOsm. For simultaneous presynaptic and postsynaptic action potential (AP) recording, the presynaptic pipette solution contained the following (mM): 110 potassium gluconate, $30 \mathrm{KCl}, 5$ EGTA, 12 disodium phosphocreatine, 10 L-glutamate, $1 \mathrm{MgCl}_{2}, 3 \mathrm{Mg}$ ATP, and $0.3 \mathrm{Na}_{2}-\mathrm{GTP}$, at $\mathrm{pH} 7.3-7.4$, adjusted with $\mathrm{KOH}$, and 315-320 $\mathrm{mOsm}$. The postsynaptic pipette solution contained the following $(\mathrm{mm})$ : 120 potassium gluconate, $30 \mathrm{KCl}, 5 \mathrm{EGTA}, 12$ disodium phosphocreatine, $1 \mathrm{MgCl}_{2}$, $3 \mathrm{Mg}$-ATP, $0.3 \mathrm{Na}_{2}$-GTP, and $1 \mathrm{~L}$-arginine, at $\mathrm{pH} 7.3-7.4$ adjusted with $\mathrm{KOH}, 315-320 \mathrm{mOsm})$. To depolymerize MTs, slices were incubated for $20-60 \mathrm{~min}$ at PT $\left(35-37^{\circ} \mathrm{C}\right)$ with vinblastine sulfate $(50$ $\mu \mathrm{M}$; Wako) dissolved in aCSF. To depolymerize F-actin, slices were incubated with latrunculin A ( $20 \mu \mathrm{M}$; Wako) at PT for $60 \mathrm{~min}$.

Fluorescence imaging. For fixed tissue imaging, the following primary antibodies were used: Anti-VGluT1 guinea pig antiserum (1:2000; AB5905, Millipore; RRID:AB_2301751); anti-synaptophysin rabbit polyclonal antiserum (1:250; catalog \#101002, Synaptic Systems; RRID: AB_887905); anti- $\alpha$-tubulin mouse monoclonal clone DM1A (1:250; catalog \#T9026, Sigma-Aldrich; RRID:AB_477593); and anti- $\beta 3$-tubulin rabbit antiserum (1:1000; product \#2200, Sigma-Aldrich; RRID: AB_262133). Secondary antibodies were goat IgG conjugated with Invitrogen Alexa Fluor 488, 568, or 647 (Thermo Fisher Scientific). For fixed tissue imaging, acute brainstem slices $(250 \mu \mathrm{m})$ were cut (see above) and fixed with $4 \%$ paraformaldehyde in $\mathrm{PBS}$ for $30 \mathrm{~min}$ at $37^{\circ} \mathrm{C}$ and overnight at $4^{\circ} \mathrm{C}$. On the following day, slices were rinsed three times in PBS, permeabilized in PBS containing 0.5\% Triton X-100 (Tx-100; Nacalai Tesque) for $30 \mathrm{~min}$ and blocked in PBS containing 3\% bovine serum albumin (BSA; Sigma-Aldrich) and 0.05\% Tx-100 for $45 \mathrm{~min}$. Slices were incubated overnight at $4^{\circ} \mathrm{C}$ with primary antibody diluted in PBS $0.05 \%$ Tx-100, $0.3 \%$ BSA. On the next day, slices were rinsed three times with PBS containing $0.05 \% \mathrm{Tx} 100$ for $10 \mathrm{~min}$ and incubated with corresponding secondary antibody diluted in PBS $0.05 \% \mathrm{Tx}-100,0.3 \% \mathrm{BSA}$ for $1 \mathrm{~h}$ at room temperature (RT). Slices were further rinsed three times in PBS $0.05 \% \mathrm{Tx}-100$ for $10 \mathrm{~min}$ and finally were washed in PBS for another 10 min. Before mounting, the nucleus was stained with Life Technologies NucBlue (Thermo Fisher Scientific) in PBS for 20 min according to manufacturer instruction. For confocal imaging, slices were then directly mounted on glass slides (Matsunami) using a mounting medium (Ibidi) and sealed using nail polish. For STED microscopy, slices were sequentially incubated in PBS containing $10 \%, 20 \%$, and $50 \%$ of $2,2^{\prime}$-thiodiethanol (TDE) for $1 \mathrm{~h}$ each, followed by washing three times in $97 \%$ TDE solution for 10 min each, and mounted on glass slides using TDE mounting reagent (Abberior).

For live imaging of silicon-rhodamine ( $\mathrm{SiR}$ )-tubulin-stained slices, acute brainstem slices $(250 \mu \mathrm{m})$ were incubated with SiR-tubulin $(1 \mu \mathrm{M}$; Cytoskeleton) at $37^{\circ} \mathrm{C}$ according to manufacturer instructions. The slices were then mounted onto a $35 \mathrm{~mm}$ Ibidi dish and immobilized using a platinum grid holder, then incubated in aCSF containing $50 \mu \mathrm{M}$ vinblastine at $37^{\circ} \mathrm{C}$. HeLa cells, cultured in DMEM $10 \%$ FBS and grown in a 35 $\mathrm{mm}$ Ibidi dish for $3 \mathrm{~d}$, were incubated with $1 \mu \mathrm{M}$ SiR-tubulin or SiR-actin at $37^{\circ} \mathrm{C}$ and $5 \% \mathrm{CO}_{2}$. Culture medium was replaced with Tyrode's solution before vinblastine or latrunculin A treatment and observation.

Confocal images were acquired on a laser scanning microscope (LSM 780 , Carl Zeiss) equipped with a Plan-apochromat $63 \times$, oil-immersion objective with a numerical aperture (NA) of 1.4 and excitation laser lines (wavelengths: 405, 488, 561, and $633 \mathrm{~nm}$ ). For quantifying fluorescence intensity levels, the region of interest was delimited to calyceal terminals, and background fluorescence was subtracted using ImageJ software. Super-resolution imaging was performed on STED $3 \times$ TCS SP8 microscope (Leica) equipped with an HC PL APO CS2 100×, 1.4 NA oilimmersion objective (Leica) and tunable white laser excitation line, and with depletion laser lines (wavelengths: 592, 660, and $775 \mathrm{~nm}$ ). STED images were deconvoluted using Huygens software (Leica). Threedimensional reconstruction of STED $3 \times$ confocal stacks was performed in Imaris 9.2 with filament tracer and measurement pro plugins (Bitplane/Oxford Instruments) to estimate the distance between identified vesicles and microtubules.

Electrophysiological recordings and data analysis. The calyx of Held presynaptic terminals and postsynaptic MNTB principal cells were visually 
identified with a $40 \times$ water-immersion objective attached to an upright microscope (BX51WI, Olympus). All experiments were performed at PT. EPSCs were evoked in MNTB principle neurons by afferent fiber stimulation using a bipolar tungsten electrode, positioned on the axon bundles halfway between the midline and MNTB region. For whole-cell recording of EPSCs, MNTB principal neurons were voltage clamped at a holding potential of $-70 \mathrm{mV}$. Postsynaptic pipettes were pulled for a resistance of 2-3M $\Omega$ and had a series resistance of 4-10 $\mathrm{M} \Omega$, which was compensated by $40-70 \%$ for a final value of $3 \mathrm{M} \Omega$. STD of EPSCs was induced by a train of 30 stimuli at $100 \mathrm{~Hz}$, and recovery from STD was monitored from EPSCs evoked by stimulations at different intervals $(0.02-30 \mathrm{~s})$. The recovery time course was fit by a double exponential function, from which fast time constants $\left(\tau_{\text {fast }}\right)$ and slow time constants $\left(\tau_{\text {slow }}\right)$ were measured.

Presynaptic membrane capacitance measurements at calyces of Held were made as previously described (Sun et al., 2002; Yamashita et al., 2005) except that recordings were made at PT in the present study instead of RT. Calyceal terminals were voltage clamped at a holding potential of $-80 \mathrm{mV}$, and single-pulse step to $+10 \mathrm{mV}$ (20 ms in duration) was applied for inducing presynaptic $\mathrm{Ca}^{2+}$ currents. The rate of endocytosis was evaluated from the $50 \%$ decay time of membrane capacitance change.

Data analysis. Electrophysiological data were acquired at a sampling rate of $50 \mathrm{kHz}$ using an EPC-10 patch-clamp amplifier controlled by PatchMaster software (HEKA), after on-line filtering at $5 \mathrm{kHz}$ and subsequently analyzed off-line using IGOR Pro 6.22 (WaveMetrics), Excel 2010 (Microsoft), Origin Pro 8.6 (Origins Laboratory), SPSS (IBM), and Prism 6 (GraphPad Software). Data fitting was performed using the leastsquares method (single or double exponential). Imaging data were analyzed using Las AF Lite (Leica), ZEN (Zeiss), and ImageJ. All values are given as the mean \pm SEM, and $95 \%$ confidence intervals on the difference of the means were considered statistically significant by two-tailed unpaired $t$ test or one-way ANOVA with a post hoc Scheffé's test $(p<$ $0.05)$.

\section{Results \\ Colocalization of microtubules with SVs at the calyx of Held presynaptic terminals}

Before addressing the functional roles of MTs in presynaptic terminals, we examined the localization of MTs in the calyx of Held presynaptic terminals in the brainstem of juvenile rats (postnatal day 13-16) using confocal and STED microscopy. After tissue fixation and permeabilization to washout-free tubulin, immunofluorescence staining of calyceal terminals, using specific antibodies against $\alpha$ - or $\beta 3$-tubulin, revealed tubulin-polymer bundles, running along axons and extending into calyceal terminals surrounding postsynaptic MNTB neurons (Fig. $1 A-C$ ). Using STED, tubulin-polymer bundles were clearly observed in proximity with SVs labeled with vesicular glutamate transporter 1 (VGluT1, Fig. $1 D, E$ ) or synaptophysin (Fig. $1 F$ ). At high magnifications, immunofluorescent tubulin signals were seen to partially overlap with those of SVs labeled with VGluT1 (Fig. 1G,H). Three-dimensional reconstruction of STED confocal stacks and analyses of SV distance from MTs indicated that 59\% of SVs are localized on or alongside the MT lattice within $100 \mathrm{~nm}$ in the whole terminal (Fig. $2 A, B$ ). In presynaptic swellings, 36\% of SVs were localized within $100 \mathrm{~nm}$ of MTs with an average \pm SEM distance of $44 \pm 2.5 \mathrm{~nm}(n=106 \mathrm{SVs})$, whereas $64 \%$ of SVs were distributed $>100 \mathrm{~nm}$ away from MTs with an average distance of $408 \pm 16 \mathrm{~nm}(n=106$; Fig. $2 B)$. These results indicate that MTs are extended and spread into calyceal swellings and suggest that MTs are partially colocalized with a subset of glutamatergic SVs in the nerve terminal.

\section{Monitoring depolymerization of microtubules in presynaptic terminals in slices}

To address the functional roles of MTs in presynaptic terminals, we depolymerized MTs by incubating slices with vinblastine at PT. The extent of MT depolymerization by vinblastine was assayed in living samples by real-time monitoring SiR-tubulin fluorescence intensity (Lukinavičius et al., 2014; Guillaud et al., 2017). After applying SiR-tubulin to a bath solution, tubulin polymers labeled with SiR were clearly visible throughout the terminals (Fig. $3 A$ ). During the incubation of slices with vinblastine $(50 \mu \mathrm{M})$, SiR-tubulin fluorescence in calyceal terminals gradually declined in intensity. After $20 \mathrm{~min}$ of treatment with vinblastine $(50 \mu \mathrm{M})$, SiR-tubulin fluorescence intensity decreased by $\sim 30 \%$, whereas it remained unchanged in untreated controls (Fig. 3B). Longer treatment further reduced the fluorescence intensity by up to $90 \%$ after $120 \mathrm{~min}$ of incubation. The depolymerizing effect of vinblastine was dose dependent (1-50 $\mu \mathrm{M}$, Fig. $3 C$ ). The MT-specific depolymerizing effect of vinblastine treatment was confirmed in a separate set of experiments using HeLa cells, where vinblastine $(50 \mu \mathrm{M})$ treatment reduced SiR-tubulin fluorescence, but had no effect on SiR-actin fluorescence, whereas the latter was reduced by incubation with latrunculin A ( $20 \mu \mathrm{M}$; data not shown). Further, to confirm the extent of MT-depolymerization by vinblastine, we measured the fluorescence intensities of tubulin isoforms in calyceal terminals in slices fixed with paraformaldehyde after $60 \mathrm{~min}$ vinblastine treatment $(50 \mu \mathrm{M})$ and immunostained with $\alpha$ - or $\beta 3$-tubulin antibody (Fig. $3 D$ ). After vinblastine treatment, both $\alpha$ - and $\beta 3$-tubulin fluorescence intensity declined by $30-40 \%$, whereas VGluT1 fluorescence remained unchanged (Fig. $3 E$ ).

\section{Depolymerization of microtubules or F-actin had no effect on basal synaptic transmission or exoendocytosis of synaptic vesicles}

Using vinblastine, we first examined whether MTs might be involved in the regulation of basal synaptic transmission at PT (Fig. $4 A)$. After vinblastine treatment $(50 \mu \mathrm{M})$ for 20-60 min, neither mean amplitude nor rise-time kinetics of EPSCs was changed. The decay time constant of EPSCs also remained unchanged. Likewise, latrunculin A treatment for $60 \mathrm{~min}$ had no effect on the amplitude or kinetics of EPSCs (Fig. 4B). Neither the frequency nor the amplitude of miniature EPSCs was affected by vinblastine or latrunculin treatment (data not shown).

We next examined whether depolymerization of MTs or F-actin might affect SV exocytosis or endocytosis, using membrane capacitance measurements at the calyx of Held presynaptic terminals (Sun et al., 2002; Yamashita et al., 2005). Exocyticendocytic changes of membrane capacitance were induced by presynaptic $\mathrm{Ca}^{2+}$ currents elicited by a $20 \mathrm{~ms}$ pulse stepped from -80 to $10 \mathrm{mV}$ at PT. Vinblastine treatment ( $50 \mu \mathrm{M}, 30-60 \mathrm{~min})$ had no effect on $\mathrm{Ca}^{2+}$ current charge $\left(Q_{\mathrm{Ca}}\right)$, magnitude of exocytosis $\left(\Delta C_{\mathrm{m}}\right)$, or endocytic kinetics (Fig. $4 C$ ). Likewise, latrunculin A treatment $(20 \mu \mathrm{M}, 60 \mathrm{~min})$ had no effect on $Q_{\mathrm{Ca}}$ or SV exocytosis-endocytosis (Fig. 4D). Thus, depolymerization of MTs or F-actin had no effect on the exocytosis-endocytosis of synaptic vesicles or basal synaptic transmission at the calyx of Held.

Like basal synaptic transmission, STD induced by a brief $(0.3 \mathrm{~s})$ train of stimulation at high frequency $(100 \mathrm{~Hz})$ was not significantly affected by vinblastine (Fig. $5 A ; p=0.1, n=10$ ) or latrunculin A (Fig. $5 B ; p=0.08, n=7$ ), although the STD magnitude showed a tendency to increase after their treatments. Likewise, the size of readily releasable vesicles (RRPs) estimated from 
A

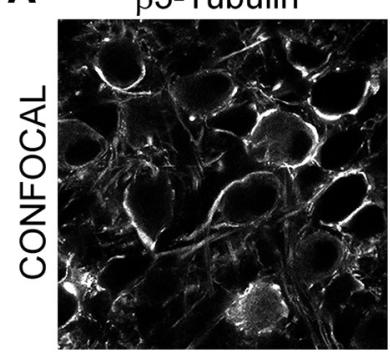

B

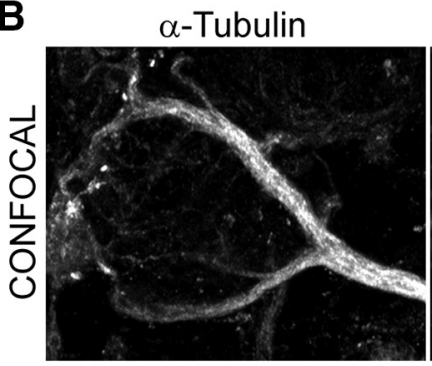

D

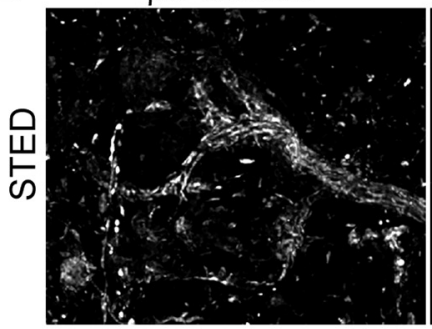

F
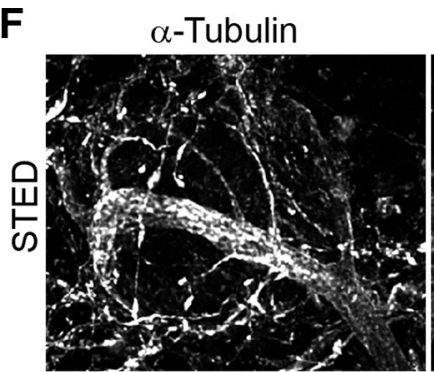

G

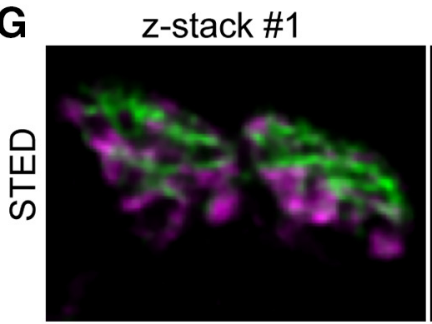

$\alpha$-Tubulin

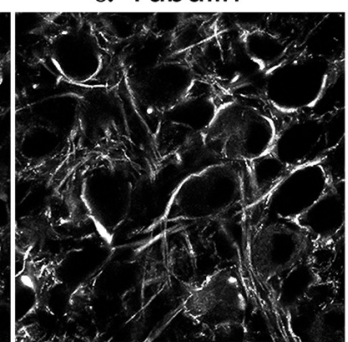

VGluT1

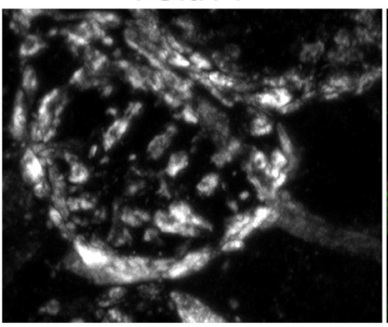

VGluT1

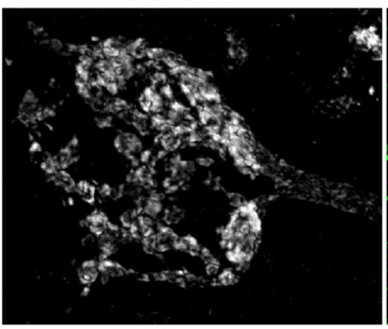

Synaptophysin

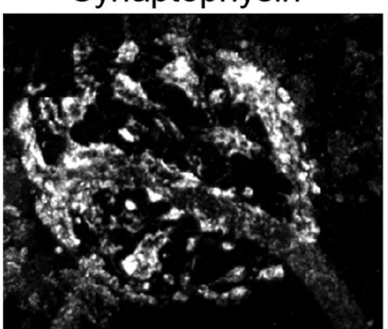

z-stack \#2

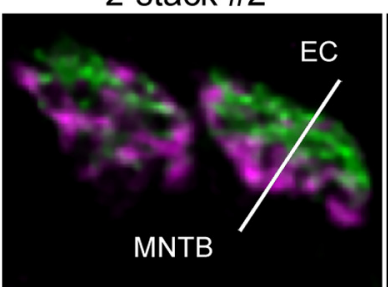

VGluT1
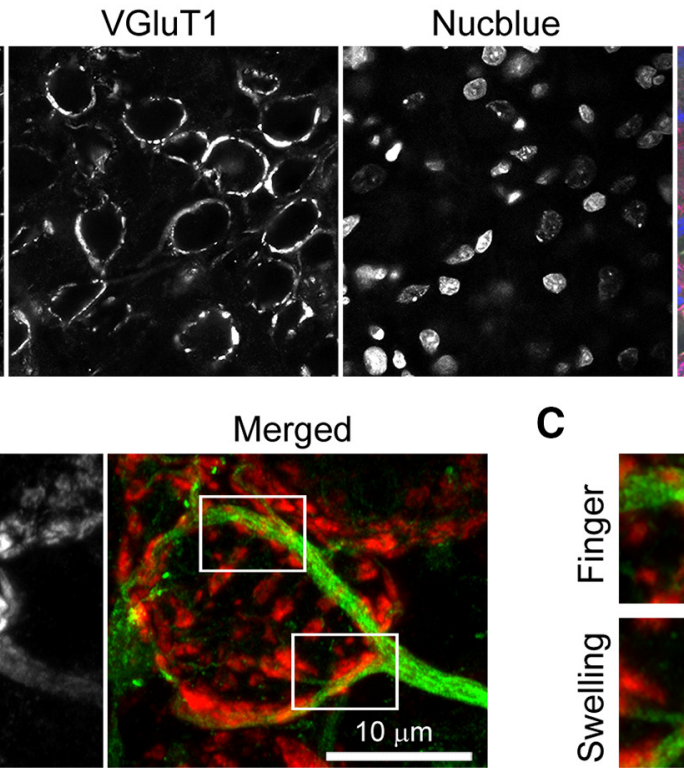

Merged

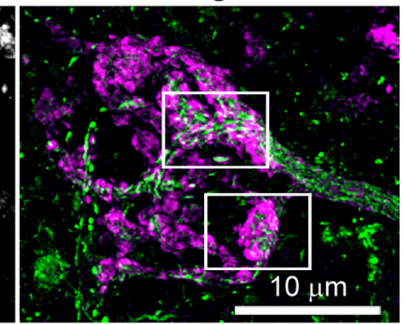

Merged

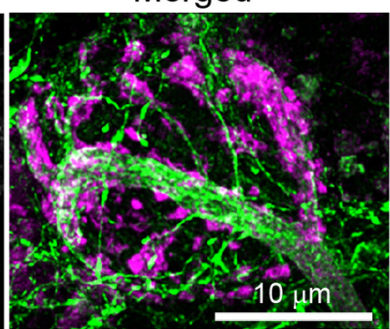

z-stack \#3

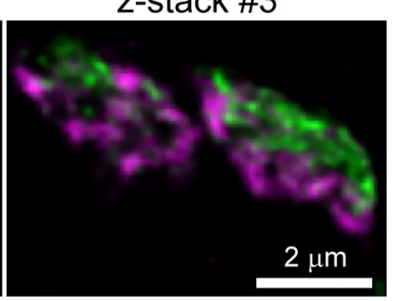

C

Merged
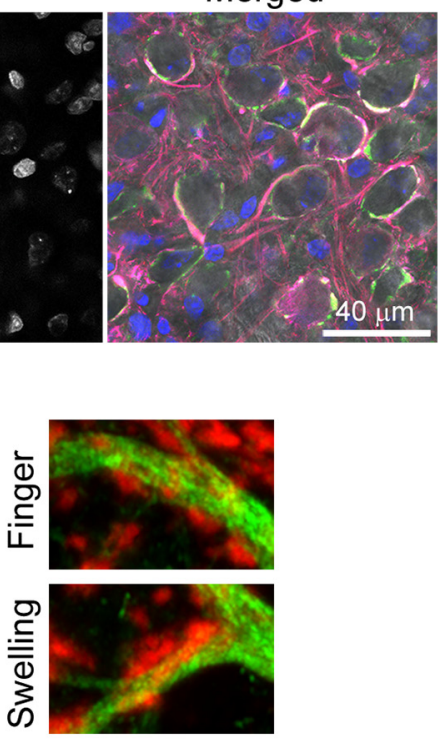

E
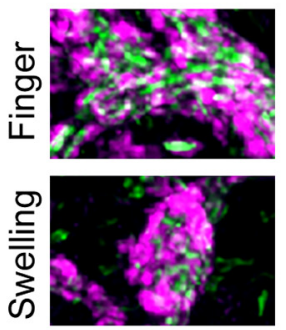

Figure 1. Localization of microtubules in the calyx of Held presynaptic terminals. A, Confocal images of $\beta 3$-tubulin, $\alpha$-tubulin, VGluT1, and Nucblue in the MNTB region of postnatal day 15 brainstem slice. $\boldsymbol{B}$, Confocal images of $\alpha$-tubulin and VGluT1 immunofluorescence, and their merged image in a calyx of Held presynaptic terminal. $\boldsymbol{C}$, Zoom-in of the area delineated in $\boldsymbol{B}$ (white boxes) showing MTs (green) and SVs (red) in presynaptic fingers and swellings. $\boldsymbol{D}$, High-resolution STED images of $\beta 3$-tubulin and VGluT1 immunofluorescence and their merged image in a calyx of Held presynaptic terminal. $\boldsymbol{E}$, Zoom-in of the area delineated in $\boldsymbol{D}$ (white boxes) showing colocalization of MTs (green) and SVs (magenta) in presynaptic fingers and swellings. $\boldsymbol{F}$, High-resolution STED image of $\alpha$-tubulin and synaptophysin in the calyx of Held presynaptic terminal. $\mathbf{G}$, High-resolution STED $z$-stack $(z=300 \mathrm{~nm})$ images of presynaptic swellings immunolabeled with $\beta 3$-tubulin (green) and VGluT1 (magenta). $\boldsymbol{H}$, Line-scan profile of the fluorescence (FI.) signal intensity in arbitrary unit (a.u.) along the line on $\mathbf{G}$ from the extracellular (EC) side to the postsynaptic (MNTB neuron) side, showing partial overlap of MTs immunolabeled with $\beta 3$-tubulin ( $\beta 3$-Tub, green) and SVs immunolabeled with VGluT1 (magenta).

cumulative EPSC amplitudes (Schneggenburger et al., 1999) showed no significant difference between slices in controls, after vinblastine treatment $(20 \mathrm{~min})$ or latrunculin A treatment (oneway ANOVA: $F_{(3,28)}=0.88, p=0.46, n=7-10$; Fig. $\left.5 C, D\right)$.
Depolymerization of microtubules or F-actin prolonged

kinetics of recovery from STD

After STD, induced by a train of nerve stimulation $(100 \mathrm{~Hz}, 0.3 \mathrm{~s})$ at PT, EPSCs underwent a biexponential recovery with a subsec- 

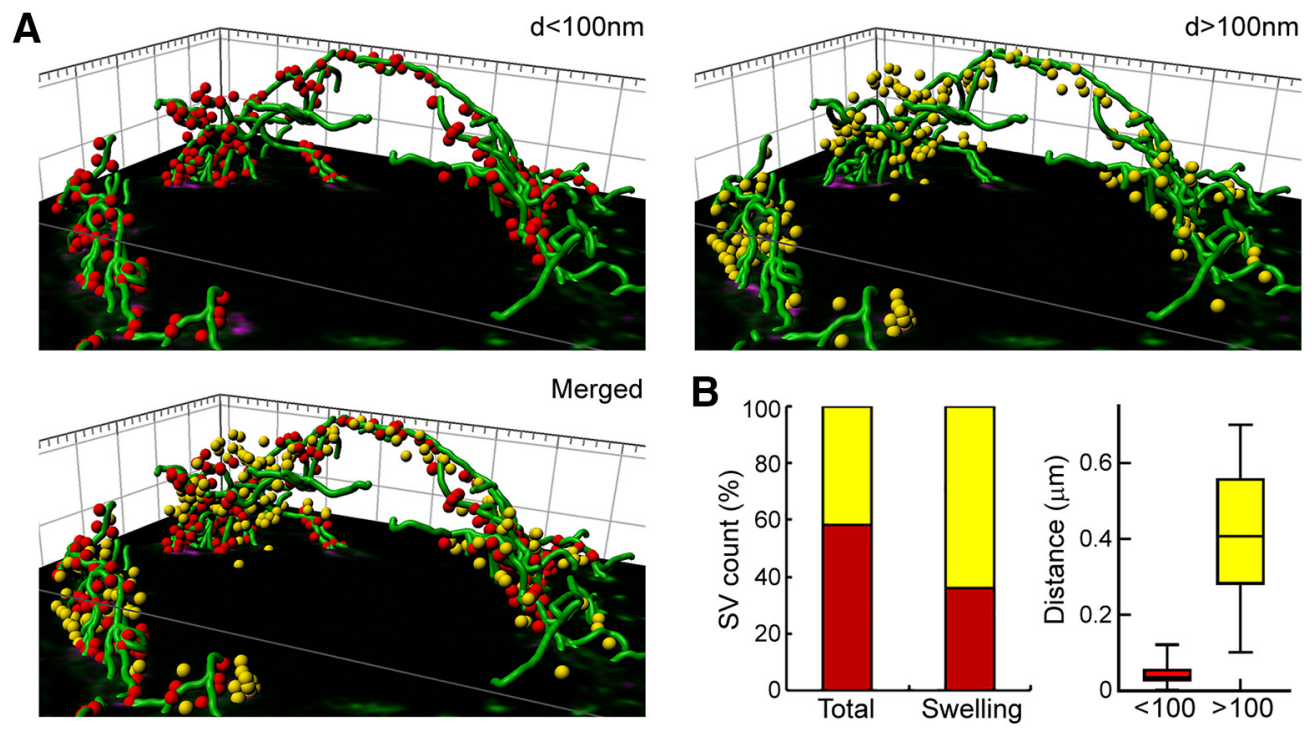

Figure 2. 3D reconstruction of SVs and MTs in a presynaptic terminal. $A, 3 D$ reconstruction of STED confocal stacks from Figure $1, D$ and $G$, showing MTs (green) and SVs (red, distance (d) $<100$ $\mathrm{nm}$, left; yellow, $d>100 \mathrm{~nm}$, right; merged, bottom left). $\boldsymbol{B}$, Bar graphs for the percentage of SVs localized at $<100 \mathrm{~nm}$ (red) or $>100 \mathrm{~nm}$ (yellow) from MTs, in the whole terminal (left) and in four swellings in the terminal (right). Box chart of the average distance estimated between SVs and MTs in presynaptic swelling for the two groups of SVs (red, $<100 \mathrm{~nm}$; yellow, $>100 \mathrm{~nm} ; n=106 \mathrm{SV}$ s for each group). Error bars indicate \pm SEM.

ond time constant $\left(\tau_{\text {fast }}\right)$ followed by a second-order time constant $\left(\tau_{\text {slow }} ;\right.$ Fig. $\left.6 A\right)$. Although vinblastine treatment for 20-60 min had no significant effect on the STD magnitude (Fig. $5 A, B$ ), it markedly and specifically prolonged the $\tau_{\text {slow }}$ (one-way ANOVA: $F_{(2,24)}=16.15, p<0.01 ; n=10$ and 7 , respectively, for 20 and 60 min of treatment) without affecting the $\tau_{\text {fast }}$ (one-way ANOVA: $\left.F_{(2,24)}=0.24, p=0.39\right)$. The magnitude of $\tau_{\text {slow }}$ prolongation by vinblastine showed a strong correlation (Pearson correlation coefficient, $r=0.98$ ) with that of MT depolymerization, assayed using SiR-tubulin (Fig. 3B) during vinblastine treatment (Fig. 6B). In clear contrast to vinblastine, latrunculin A treatment $(20 \mu \mathrm{M}, 60 \mathrm{~min})$ specifically prolonged the fast recovery time constant (two tailed unpaired $t$ test: $p<0.05, n=7$ cells) with no effect on the slow time constant (Fig. 6C). The percentage of $\tau_{\text {fast }}$ relative to $\tau_{\text {slow }}$ was essentially the same with or without treatment with vinblastine or latrunculin A (Fig. 6D).

In various cell systems, MTs and F-actin reportedly cross talk functionally (Malorni et al., 2003; Etienne-Manneville, 2004; Arnette et al., 2016). To examine whether this might be the case for the recovery of EPSCs from STD, we cotreated slices with vinblastine and latrunculin A. After $60 \mathrm{~min}$ incubation of slices with vinblastine $(50 \mu \mathrm{M})$ and latrunculin A $(20 \mu \mathrm{M})$, recovery from STD was significantly prolonged, both at the fast and slow components (Fig. $6 E$ ), as expected from the summed effect of vinblastine or latrunculin A (Fig. 6A,C). Thus, MTs and F-actin differentially contribute to slow and fast recoveries of EPSCs from STD without cross talk.

\section{Physiological roles of MTs and F-actin in high-}

\section{frequency neurotransmission}

Depolymerization of MTs or F-actin had no significant effect on basal synaptic transmission (Fig. 4) or STD (Fig. 5), but significantly prolonged the recovery of EPSCs from STD (Fig. 6). These results suggest that cytoskeletal filaments may play essential roles in the maintenance of long-lasting high-frequency neurotransmission. To test this, we first evoked EPSCs at $100 \mathrm{~Hz}$ for $45 \mathrm{~s}$ (Fig. $7 A$ ). EPSCs continuously underwent a depression during stimulation, and the magnitude of depression at $40-45 \mathrm{~s}$ was signifi- cantly greater in slices treated with vinblastine (one-way ANOVA: $50 \mu \mathrm{M}, 20 \mathrm{~min} ; n=10, p<0.01$ ) or latrunculin A (one-way ANOVA: $20 \mu \mathrm{M}, 60 \mathrm{~min} ; n=8, p<0.05$ ) relative to controls $(n=12$; Fig. $7 B, C)$.

To evaluate the impact of EPSC depression on synaptic neurotransmission, we next made simultaneous whole-cell recordings of presynaptic and postsynaptic APs, elicited by a presynaptic stimulation at $100 \mathrm{~Hz}$ at PT (Fig. 7D). Compared with similar experiments at RT (Eguchi et al., 2017), the fidelity of neurotransmission (post-AP/pre-AP) remained higher, close to $100 \%$ for $50 \mathrm{~s}$, at PT (Fig. $7 E, F)$. Vinblastine treatment ( $50 \mu \mathrm{M}$, $20 \mathrm{~min}$ ) impaired the fidelity of neurotransmission by increasing the number of AP failures in postsynaptic neurons $(n=6, p<$ 0.01 , one-way ANOVA). Latrunculin A treatment $(20 \mu \mathrm{M}, 60$ min) also impaired the fidelity ( $n=5, p<0.05$, one-way ANOVA), but to a lesser extent than vinblastine treatment (Fig. $7 E, F)$. These results suggest that presynaptic cytoskeletal filaments, MTs and F-actin, significantly contribute to the maintenance of high-frequency neurotransmission.

\section{Discussion}

The presence and functional role of MTs in the presynaptic terminal have been a matter of debate (Bodaleo and GonzalezBillault, 2016). In the calyx of Held terminals of rat brainstem, we confirmed our previous observation in cultured calyceal terminals (Guillaud et al., 2017) that axonal MTs are extended into terminal swellings and further found, using STED microscopy, that they are partially colocalized with SVs. These results agree with previous EM studies indicating the presence of tubulin polymers at the frog NMJ (Gray, 1978, 1983; Hirokawa et al., 1989).

To address whether MTs play a role in synaptic transmission, we depolymerized MTs using vinblastine after estimating its potency using SiR-tubulin live imaging. When MTs in calyceal terminals were depolymerized with vinblastine, the recovery of EPSCs from STD was prolonged, exclusively at the slow component of biexponential recovery. This slow component was resistant to F-actin depolymerization by latrunculin A (Fig. 6C) as previously reported (Sakaba and Neher, 2003), and it is distinct 
A

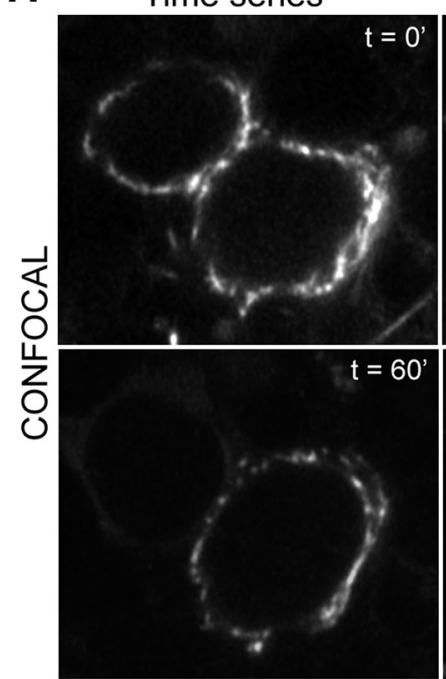

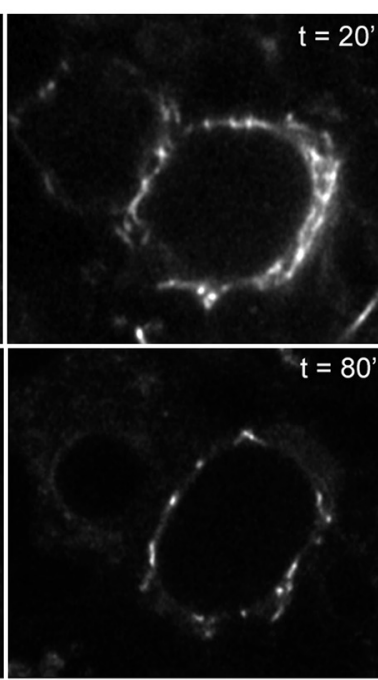

D

\section{D $\quad \beta 3-T u b u l i n$}
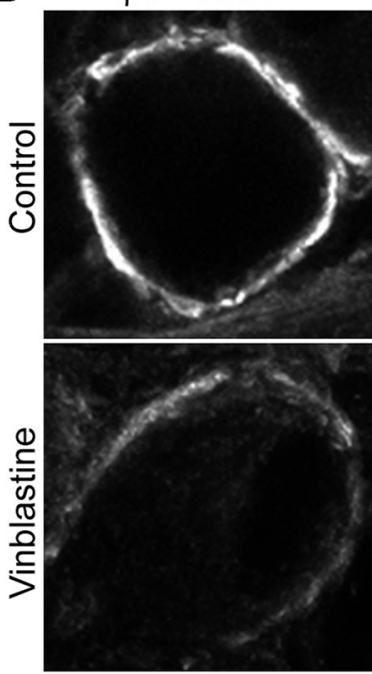

$\mathbf{E}$

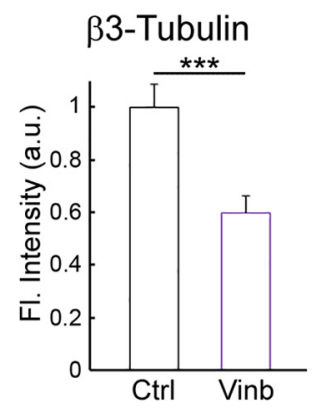

$\alpha$-Tubulin
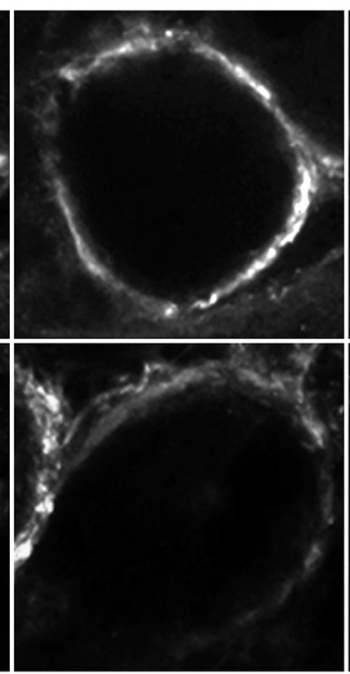

$\alpha$-Tubulin

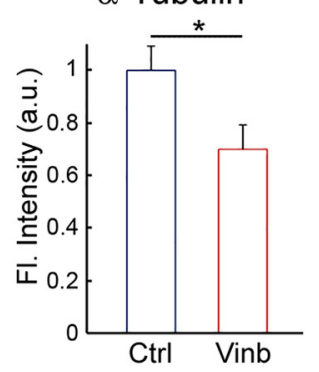

B
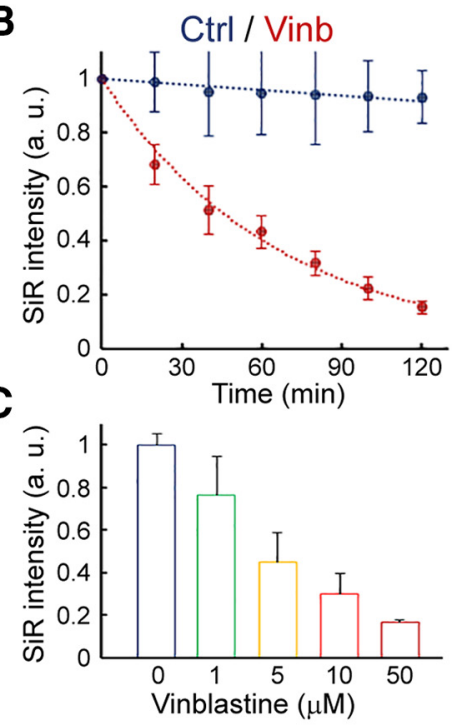
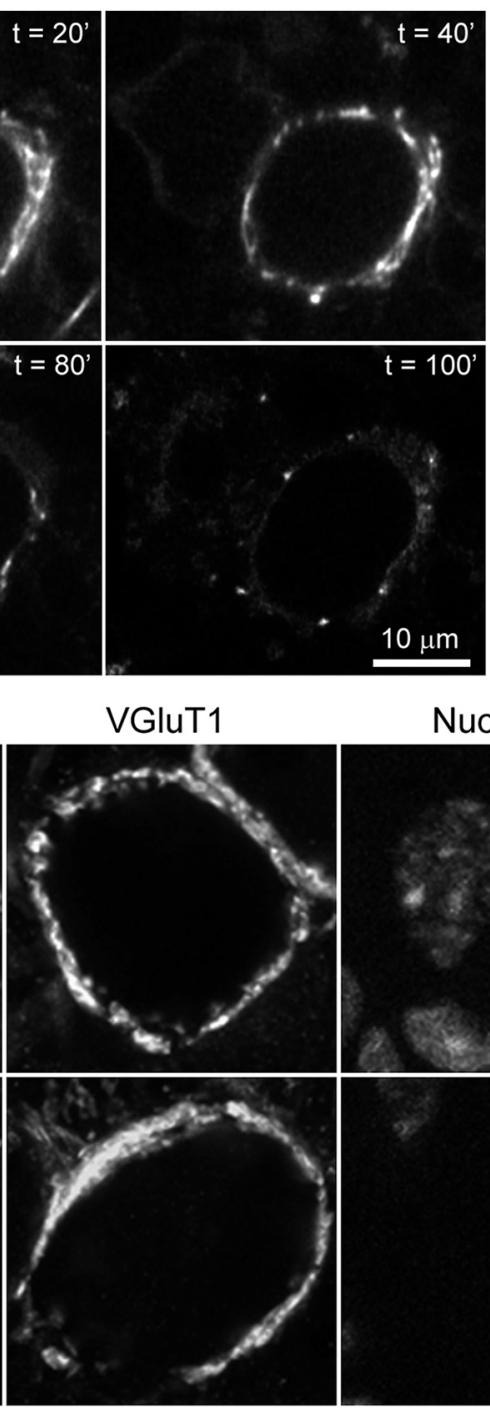

Nucblue
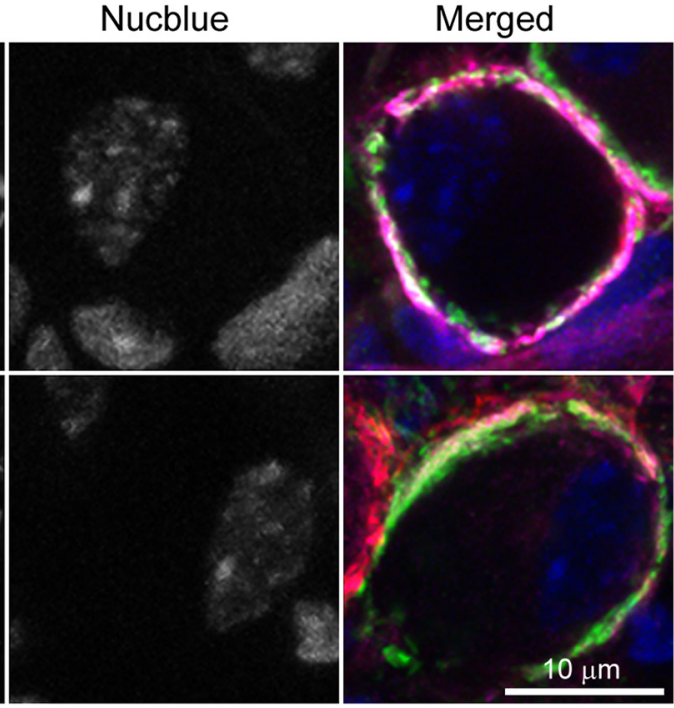

VGluT1

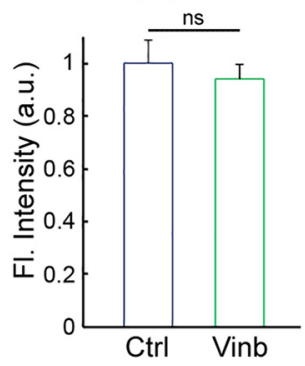

Figure 3. Depolymerization of microtubules in calyx of Held presynaptic terminals. A, Real-time imaging of MTs labeled with SiR-tubulin in live slices, indicating progressive depolymerization before $(t=0)$ and during $(t=20-100 \mathrm{~min})$ vinblastine treatment $(50 \mu \mathrm{m})$ at PT. B, Time plots showing normalized SiR-tubulin fluorescence intensity with (red) or without (blue) vinblastine treatment $(50 \mu \mathrm{M}$ ) from three independent experiments ( $n=9$ cells from 3 slices of 3 animals). $C$, Bar graphs showing the dose-dependent effect of vinblastine on SiR-tubulin fluorescence intensity after $2 \mathrm{~h}$ of treatment at PT ( $n=3$ cells). $\boldsymbol{D}$, Confocal images of calyces of Held terminals in fixed slices immunolabeled with $\beta 3$-tubulin, $\alpha$-tubulin, and VGluT1, without (top panels) or after (bottom panels) vinblastine $(50 \mu \mathrm{m})$ treatment for $60 \mathrm{~min}$ at PT. E, Quantification of fluorescence intensity of $\beta 3$-tubulin [control (Ctrl), $n=20$ from 3 slices of 3 animals; vinblastine (Vinb), $n=23 ; p=$ 0.0008), $\alpha$-tubulin (Ctrl, $n=20 ; \operatorname{Vinb}, n=23 ; p=0.023$ ), and VGluT1 (Ctrl, $n=31 ; \operatorname{Vinb}, n=28 ; p=0.589$ ), without treatment (Ctrl) or after vinblastine treatment (Vinb). Unpaired Student's $t$ test: ${ }^{*} p<0.05 ;{ }^{* * *} p<0.001$. ns, Not significant; Ctrl, control; Vinb, vinblastine. Error bars indicate \pm SEM.

from the recovery of the slow releasing pool (Sakaba and Neher, 2001). The time constant of this slow recovery component $(\sim 2 \mathrm{~s}$ at PT) is much faster than that of SV refilling with transmitter glutamate ( $\sim 7 \mathrm{~s}$ at PT; Hori and Takahashi, 2012), suggesting that it reflects the replenishment of SVs already filled with transmitter from reserve pool (RP) to RRP. Vinblastine slowed SV replenishment from RP to RRP (Fig. 6A) but did not significantly affect RRP size during a brief train stimulation (Fig. $5 C, D$ ). However, during a long train of stimulation, vinblastine significantly depressed EPSC amplitude presum- 
A
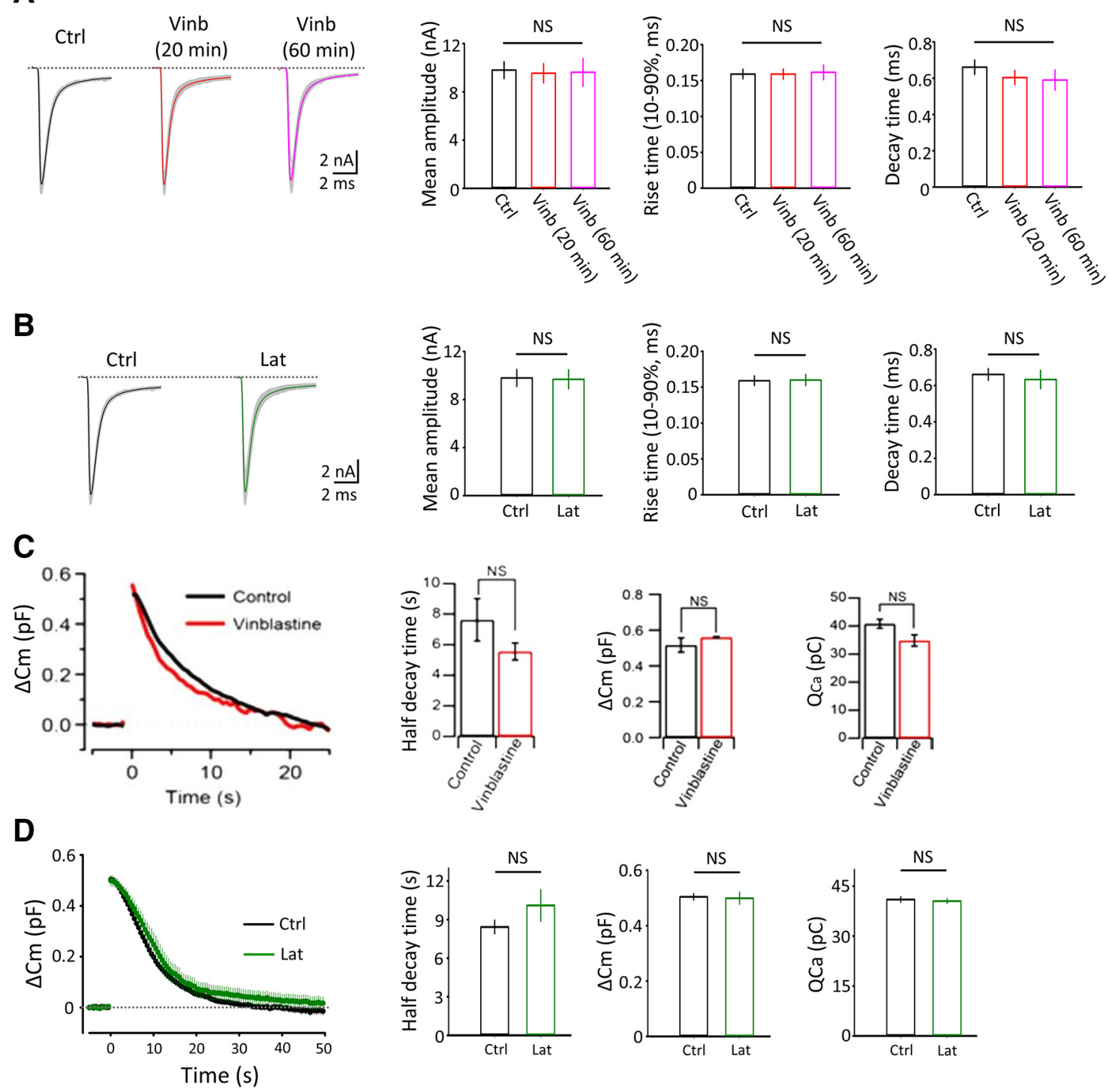

Figure 4. No significant effect of vinblastine or latrunculin A treatment on basal synaptic transmission and exocytosis-endocytosis of synaptic vesicles. $A$, EPSCs with or without vinblastine treatment ( $50 \mu \mathrm{M}, 20$ or $60 \mathrm{~min}$ ). Sample traces, control EPSCs without vinblastine treatment, and those after 20 (red) or 60 (pink) min vinblastine treatment. Bar graphs indicate mean amplitude (left), 10-90\% rise time (middle), and decay time constant (right) of EPSCs without vinblastine (black) or 20 or 60 min after vinblastine treatment. There was no significant difference in these parameters of EPSCs between vinblastine-treated and untreated slices (one-way ANOVA: $\left.F_{(2,24)}=0.76, p=0.09\right) . B$, EPSCs with or without latrunculin A treatment (20 $\mu \mathrm{m}, 60$ min). Bar graphs indicate mean amplitude (left), $10-90 \%$ rise time (middle), and decay time constant (right) of EPSCs with (green) or without (black) latrunculin A treatment. No significant difference. C, Membrane capacitance changes $\left(\Delta C_{m}\right)$ induced by $\mathrm{Ca}^{2+}$ currents (data not shown) evoked in calyceal terminals at PT in slices treated with vinblastine $(50 \mu \mathrm{m}, 30-60 \mathrm{~min}$ at PT, $n=5$ terminals) with (red trace) or without ( $n=4$, black trace) vinblastine treatment (superimposed). Bar graphs summarize endocytic half-decay time (left), exocytic magnitude $\left(\Delta C_{\mathrm{m}}\right.$, middle), and $Q_{c a}$ (right) with or without vinblastine treatment. No significant difference with $p=0.25,0.37$, and 0.054 for endocytic half-decay time, exocytic magnitude, or $\mathrm{Ca}^{2+}$ current charge, respectively. $\mathbf{D}$, Membrane capacitance changes in slices treated with latrunculin A ( $20 \mu \mathrm{m}, 60$ min at PT, $n=6$ terminals) with (green) or without ( $n=7$ terminals, black) latrunculin A treatment (superimposed). Bar graphs summarize endocytic half-decay time (left), exocytic magnitude $\left(\Delta C_{\mathrm{m}}\right.$, middle), and $Q_{\mathrm{ca}}$ (right) with or without latrunculin $A$ treatment. No significant difference with $p=0.07,0.35$, and 0.12 , respectively, for endocytic decay time, exocytic magnitude, and $\mathrm{Ca}^{2+}$ current charge (two-tailed unpaired $t$ test). NS, No significant difference; Ctrl, Control; Vinb, vinblastine; Lat, latrunculin A. Error bars indicate \pm SEM.

ably because of RRP depletion (Fig. $7 A-C$ ), thereby blocking neurotransmission (Fig. $7 D-F$ ).

The molecular mechanism underlying this slow recovery component is unknown, except for its dependence on GTP (Takahashi et al., 2000). Several possibilities may be conceived for the role of MTs in this SV transport. First, MTs may directly transport SVs to release sites, although MTs are selectively involved in long-distance SV movements in cultured calyceal terminals (Guillaud et al., 2017). Second, mitochondria, which are transported along MTs (Hirokawa et al., 2010; Melkov and Abdu, 2018), may provide ATP close to SVs to promote their move- ment. However, this is unlikely since, during simultaneous presynaptic and postsynaptic AP recording (Fig. $7 D-F$ ), the presynaptic pipette contained $3 \mathrm{~mm}$ ATP, which will hold intraterminal ATP concentration (Pusch and Neher, 1988), but vinblastine impaired transmission fidelity. In fact, in cultured hippocampal synapses, ATP concentration in presynaptic boutons is reportedly uniform between boutons with or without mitochondria, implying that ATP rapidly diffuses through axons (Pathak et al., 2015). Third, SVs tethered to MTs in the terminal (Hirokawa et al., 1989) may serve as a reserve pool for SV reuse, and tethered SVs may be scattered after MT depolymerization, 
thereby reducing the number of SVs available for recycling reuse. The molecular mechanism of this SV transport underlying the recovery of transmission after STD remains to be studied.

As reported previously at the calyx of Held at RT (Sakaba and Neher, 2003; Lee et al., 2012) and at cerebellar synapses at or near PT (Miki et al., 2016), the fast component of recovery from STD was prolonged by F-actin depolymerization with latrunculin at the calyx of Held at PT (Fig. 6C). At this synapse at RT, the fast recovery component is clearly observed when a large $\mathrm{Ca}^{2+}$ influx was induced, by high-frequency stimulation (Wang and Kaczmarek, 1998, 100 Hz, 0.5 s; Lipstein et al., 2013, 100-300 Hz, $0.1 \mathrm{~s}$ ) or by presynaptic depolarization under voltage clamp with a square pulse (Sakaba and Neher, 2001; Lipstein et al., 2013; $50 \mathrm{~ms}$ ) in the presence of $\mathrm{K}^{+}$channel blockers, whereas it is absent when EPSCs were evoked at low frequency $(10 \mathrm{~Hz}$; Takahashi et al., 2000) or at high frequency, but without $\mathrm{K}^{+}$channel blockers (Wang and Kaczmarek, 1998, 100 Hz; Mahfooz et al., 2016, $300 \mathrm{~Hz}$ ). At PT, however, the fast recovery component was prominent after $100 \mathrm{~Hz}$ stimulation in the absence of $\mathrm{K}^{+}$channel blocker (Fig. 6). The fast recovery component after STD reportedly depends on $\mathrm{Ca}^{2+}$ (Wang and Kaczmarek, 1998), calmodulin (Sakaba and Neher, 2001), Munc13-1 (Lipstein et al., 2013), as well as F-actin (Sakaba and Neher, 2003) and intersectin (Sakaba et al., 2013), and it is abolished when endocytosis is blocked (Hosoi et al., 2009). The fast recovery component is thought to reflect superpriming of SVs (Lee et al., 2012; Miki et al., 2016) through release site clearance (Hosoi et al., 2009; Hua et al., 2013) or/and fast SV replenishment (Lipstein et al., 2013).

Various roles of F-actin in the nerve terminal have been reported. A negative regulatory role of F-action on exocytic SV fusion is reported at cultured hippocampal synapses (Morales et al., 2000), but, at the calyx of Held, F-actin depolymerization had no effect on the magnitude of SV exocytosis or EPSC amplitude (Fig. 4). Genetic ablation of F-actin reportedly blocks both clathrin-dependent and -independent endocytosis (Wu et al., 2016). Pharmacological experiments can lead to controversial results due to the variable accessibility of drugs to targets, whereas the gene knock-out approach has disadvantages of compensatory and secondary effects (Wu et al., 2016). In the present study, pharmacological depolymerization
A
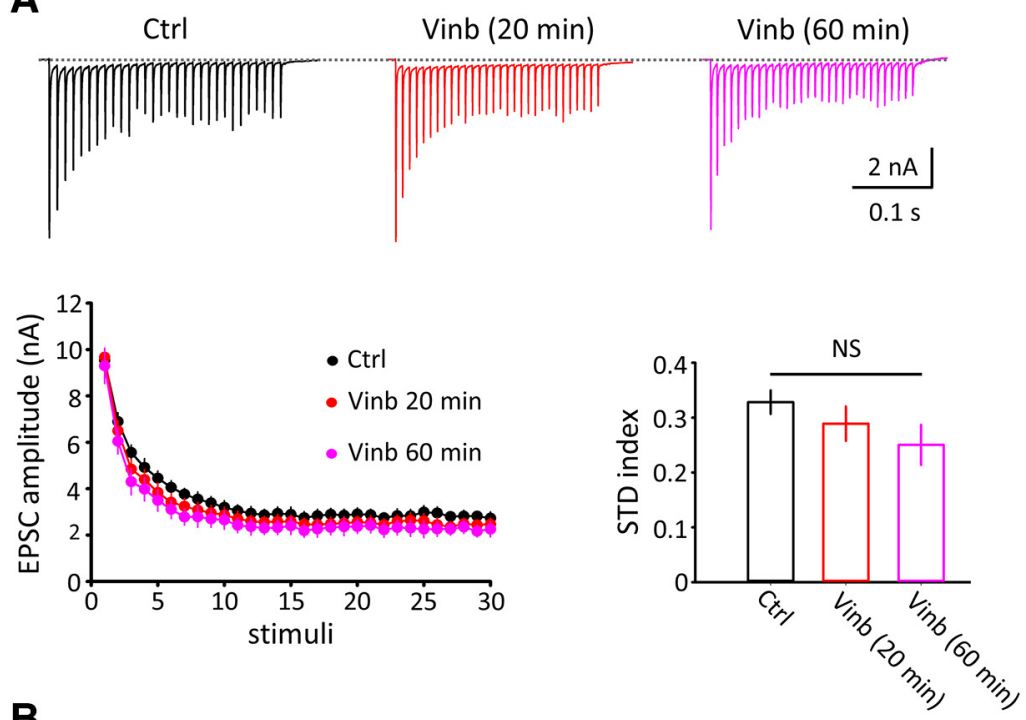

B

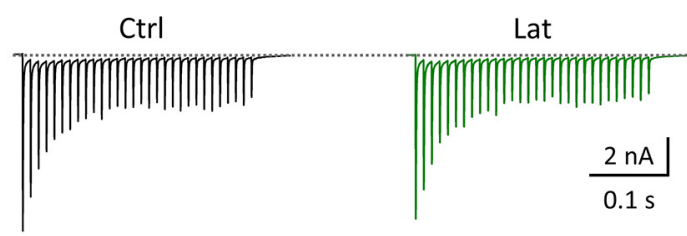

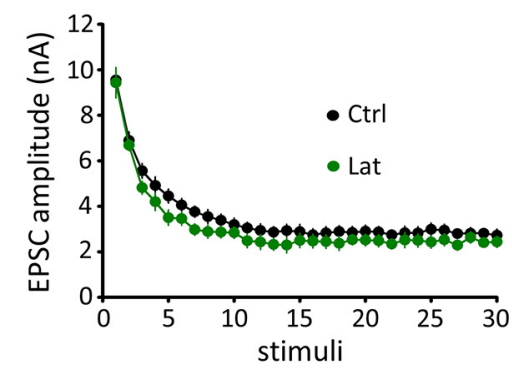

C

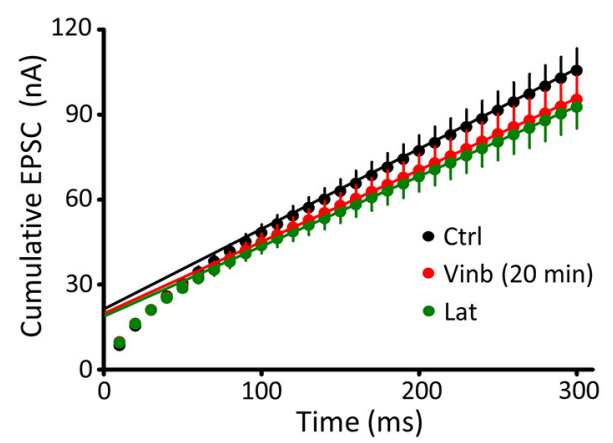

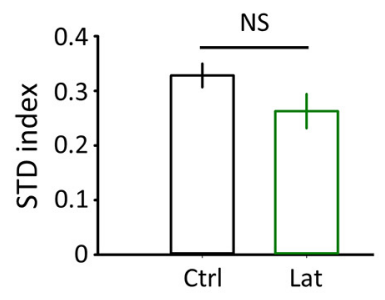

D

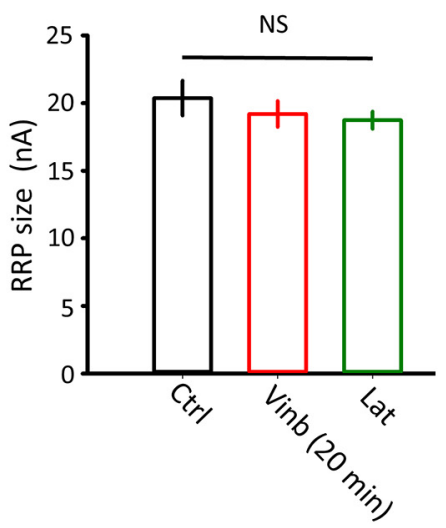

Figure 5. Depolymerization of MTs or F-actin had no effect on STD, but differentially prolonged the recovery of EPSCs from STD. $A$, Top traces, STD induced by a train of $100 \mathrm{~Hz}(\times 30)$ stimulation, with (red) or without (black) vinblastine treatment $(50 \mu \mathrm{m}, 20$ or $60 \mathrm{~min}$ ). Time plots in bottom left show STD with or without vinblastine treatment (superimposed). Bar graphs indicate the magnitude of STD measured by averaging the last 10 EPSC amplitudes divided by the initial EPSC amplitude in the train. № significant difference between them (NS, one-way ANOVA: $F_{(2,24)}=1.35, p=0.12$ ). $\boldsymbol{B}$, As in $\boldsymbol{A}$, except that slices were treated with latrunculin $\mathrm{A}(20 \mu \mathrm{M}, 60 \mathrm{~min}$ ) instead of vinblastine. No significant difference ( $p=0.08$, two-tailed unpaired $t$ test). C, Cumulative EPSC amplitude plots during a $100 \mathrm{~Hz}$ train in control (Ctrl; black), vinblastine (Vinb; $50 \mu \mathrm{M}, 20 \mathrm{~min}$, red), latrunculin A (Lat; $20 \mu \mathrm{M}$, $60 \mathrm{~min}$; green). The last 10 data points were used for fitting with linear regression lines (dashed lines) and extrapolated to $0 \mathrm{~ms}$ to estimate the RRP size of synaptic vesicles. $\boldsymbol{D}$, Mean RRP size estimated in Ctrl (20.4 $\pm 0.98 \mathrm{nA}, n=10)$, Vinb $(19.2 \pm 0.96 \mathrm{nA}, n=$ 10), Lat (18.7 $\pm 0.82 \mathrm{nA}, n=7$ ). There was no significant difference (NS) among them (one-way ANOVA: $F_{(3,28)}=0.88, p=$ 0.46). Error bars indicate \pm SEM. 
A

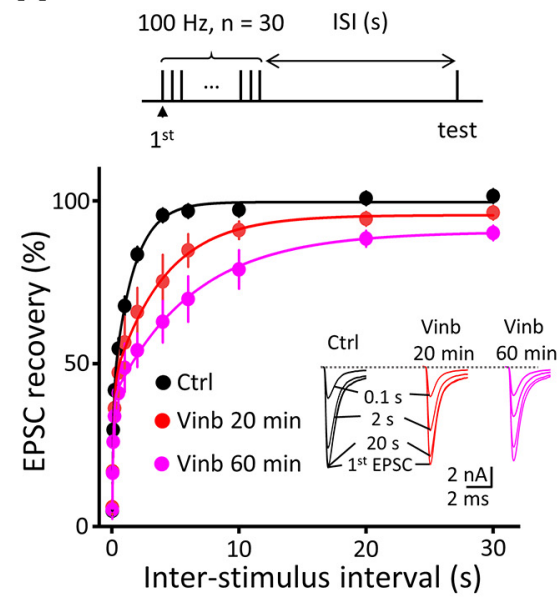

C

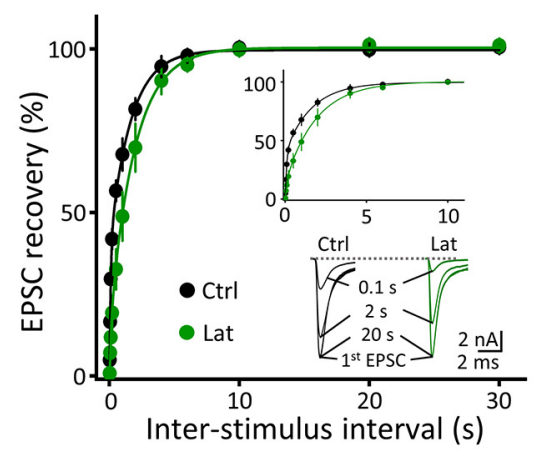

E

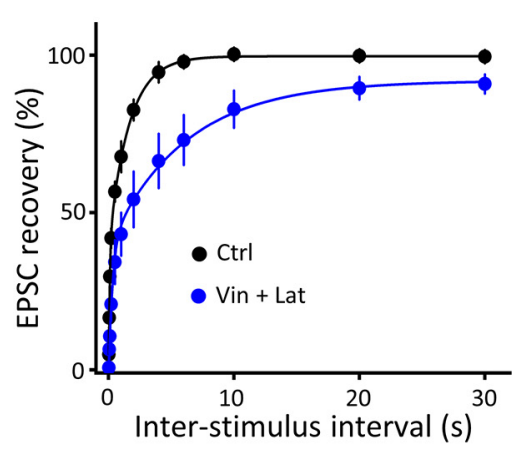

B

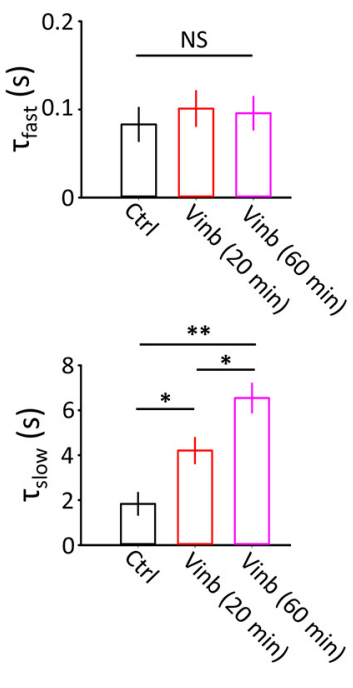

D
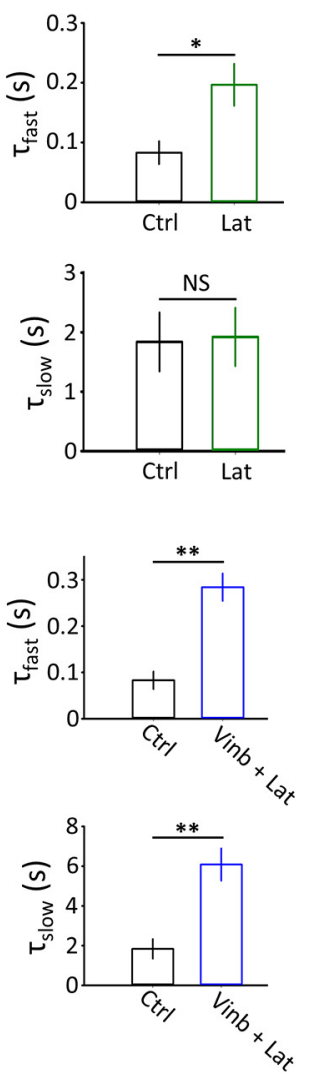

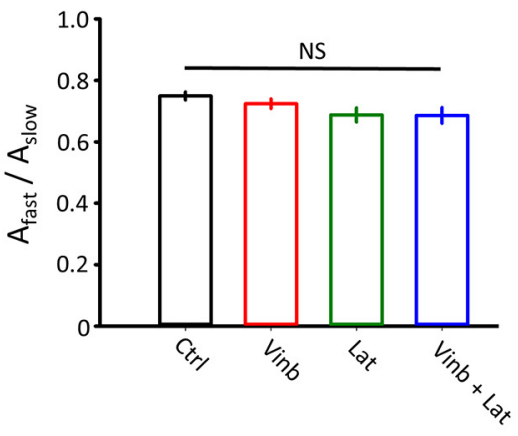

Figure 6. Depolymerization of MTs or F-actin differentially prolonged the recovery of EPSCs from STD. A, Percentage recovery of EPSC amplitude from STD at different interstimulus intervals (stimulation protocol on the top). Sample traces of EPSCs at different interstimulus intervals (ISI) are shown in inset (superimposed); 20 min (red) or 60 min (pink) after vinblastine treatment (Vinb) or without vinblastine treatment (Ctrl; black). Bar graphs in top and bottom indicate $\tau_{\text {fast }}$ and slow $\tau_{\text {slow }}$ respectively, after 20 min $(n=10)$ or 60 min ( $n=$ 7) of treatment with vinblastine or without vinblastine treatment $(n=10)$. Vinblastine treatment specifically prolonged $\tau_{\text {slow. }}{ }^{*} p<0.05,{ }^{* *} p<0.01$ (one-way ANOVA). $\boldsymbol{B}$, Correlation between the percentage of MT depolymerization (ordinate; data from Fig. 3B) and $\tau_{\text {slow }}$ values after different lengths of vinblastine treatment for $20 \mathrm{~min}(n=10), 40 \mathrm{~min}(n=5)$, and $60 \mathrm{~min}(n=7)$. The slope coefficient $(r)$ of a linear regression line is 0.98 . C, Latrunculin A treatment (Lat; $20 \mu \mathrm{m}, 60 \mathrm{~min}$; green) specifically prolonged $\tau_{\text {fast }}$ from STD, as shown with the expanded time scale in the top inset. EPSCs during recovery from STD without (black) or with latrunculin A (green) treatment are shown in the bottom inset (superimposed). Bar graphs indicate the $\tau_{\text {fast }}$ and $\tau_{\text {slow }}$ in top and bottom, respectively, with (green, $n=7$ ) or without (black, $n=10$ ) latrunculin A treatment. ${ }^{*} p<0.05$ (two tailed unpaired $t$ test). $\boldsymbol{D}$, Relative ratio of fast and slow components $\left(A_{\text {fast }} / A_{\text {slow }}\right)$ estimated for $C \operatorname{trl}(0.75 \pm 0.01, n=10$, black), Vinb $(0.72 \pm 0.02, n=10$, red), Lat $(0.69 \pm 0.02, n=7$, green $)$, and Vinb $(50 \mu \mathrm{M})+$ Lat (20 $\mu \mathrm{M}, 60 \mathrm{~min} ; 0.69 \pm 0.03, n=5$, blue). No significant difference (NS) among them (one-way ANOVA: $\left.F_{(3,28)}=2.81, p=0.06\right)$. $E$, Cotreatment of slices with vinblastine (50 $\mu \mathrm{M}$ ) and latrunculin A $(20 \mu \mathrm{m})$ for $60 \mathrm{~min}$ (blue) prolonged the recovery of EPSCs from STD at both fast and slow recovery components (bar graphs). The magnitudes of prolongation of $\tau_{\text {fast }}$ and $\tau_{\text {slow }}$ are similar to those of individual drug treatments (two-tailed unpaired $t$ test, $p=0.27$ ). Error bars indicate \pm SEM. 
A

Ctrl

Vinb

Lat

$0-1 \mathrm{~s}$
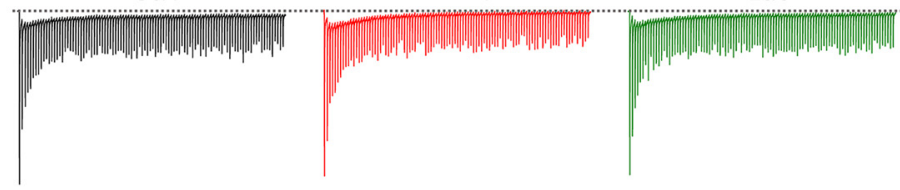

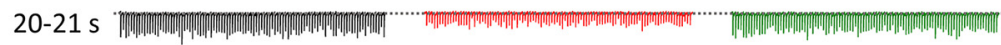

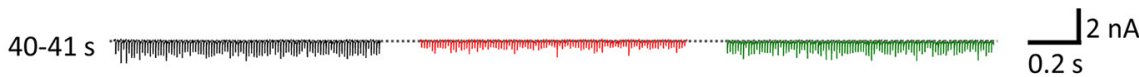

B

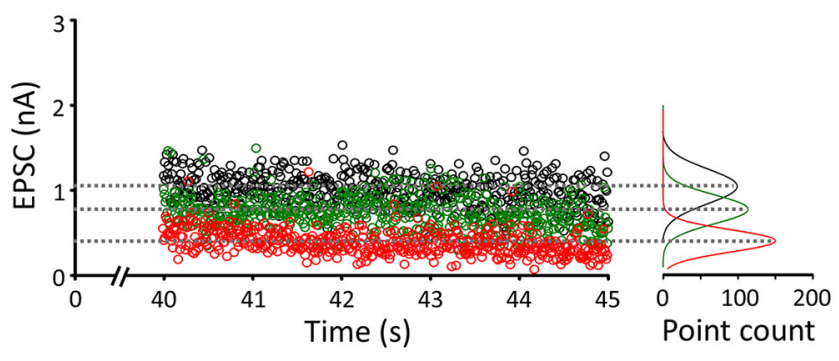

C

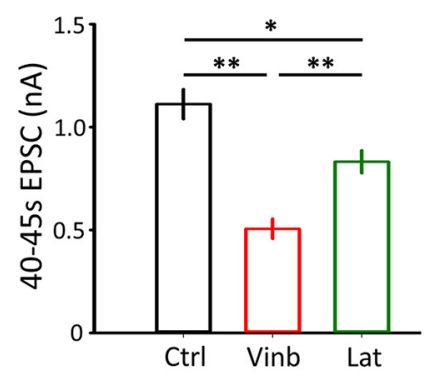

D

Ctrl

Pre AP

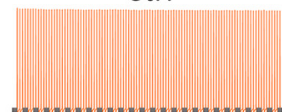

Vinb

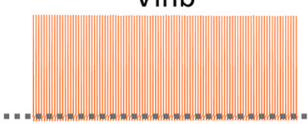

Lat

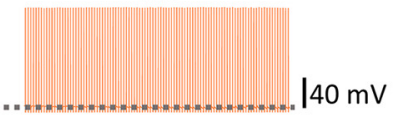

Post AP

$1-2 \mathrm{~s}$

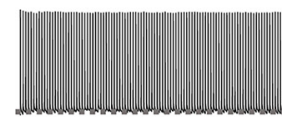

||1

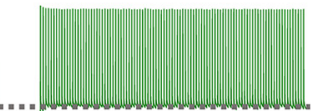

$25-26 s$

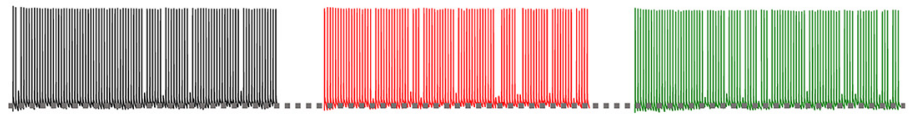

$49-50 s$

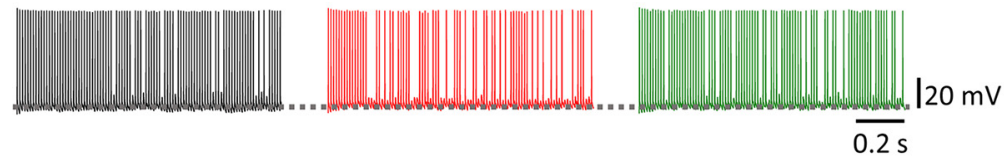

E

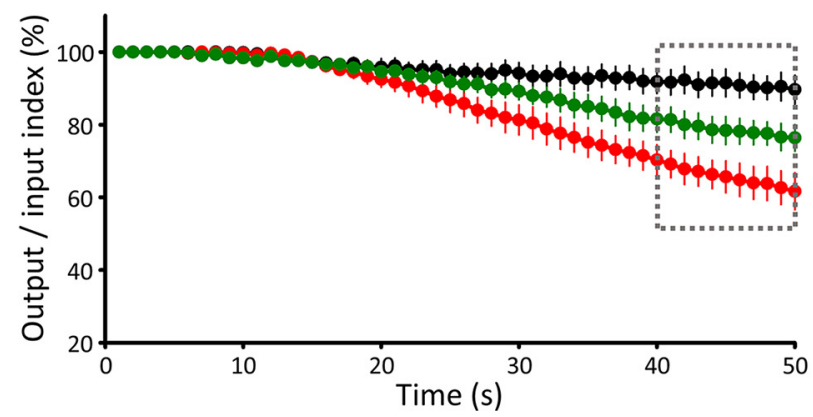

$\mathbf{F}$

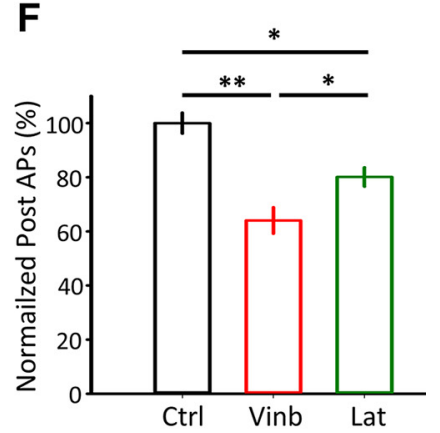

Figure 7. Presynaptic cytoskeletal filaments contribute to the maintenance of the fidelity of high-frequency neurotransmission. $A$, EPSCs evoked at PT by a train of stimulation at $100 \mathrm{~Hz}$ at $0-1$ $\mathrm{s}$ (top traces), 20 -21 s (middle), and $40-41 \mathrm{~s}$ (bottom) in control (Ctrl; black), after vinblastine treatment (Vinb; $50 \mu \mathrm{m}$; red), or latrunculin A treatment (Lat; $20 \mu \mathrm{m}, 60 \mathrm{~min} ;$ green). Stimulation artifacts are truncated. $\boldsymbol{B}$, EPSC amplitudes at $40-45 \mathrm{~s}$ with their distributions (right graphs). $C$, Bar graphs of mean EPSC amplitudes at $40-45 \mathrm{sin}$ Ctrl $(1.11 \pm 0.07 \mathrm{nA}, n=12, \mathrm{black})$, Vinb $(0.5 \pm$ $0.02 \mathrm{nA}, n=10$, red), and Lat $\left(0.83 \pm 0.05 \mathrm{nA}, n=8\right.$, green). Significant difference in EPSC amplitudes among them $\left(F_{(2,27)}=20.04, p<0.01\right.$, one-way ANOVA). ${ }^{*} p<0.05,{ }^{* *} p<0.01 . \boldsymbol{D}$, Presynaptic APs elicited at $100 \mathrm{~Hz}$ at PT (top raw traces). Postsynaptic APs evoked by presynaptic APs at $1-2 \mathrm{~s}$ (second raw trace), $25-26 \mathrm{~s}$ (third raw trace), and $49-50 \mathrm{~s}$ (bottom raw trace) after stimulation onset, without drug treatment (left columns, control; black traces), after vinblastine treatment ( $50 \mu \mathrm{m}, 20 \mathrm{~min}$, middle columns; red), or after latrunculin A treatment (20 $\mu \mathrm{M}$, $60 \mathrm{~min}$, right columns; green). $\boldsymbol{E}$, The fidelity of neurotransmission evaluated from the ratios of output AP number/input AP number (ordinate) in controls (black), after vinblastine treatment (red), or after latrunculin A treatment (green). $\boldsymbol{F}$, Bar graphs indicating the fidelity of neurotransmission at $40-50 \mathrm{~s}$ after $100 \mathrm{~Hz}$ stimulation onset. Significant difference (one-way ANOVA: $\left.F_{(2,14)}=18.51, p<0.01\right)$ in transmission fidelity between vinblastine-treated samples $(n=6)$ and controls $(n=6$; one-way ANOVA with post hoc $S c h$ effé's tests, $p<0.01$ ), between latrunculin A-treated samples $(n=5)$ and controls ( $n=6$; one-way ANOVA with post hoc Scheffé's tests, $p<0.05)$, and between vinblastine-treated samples $(n=$ 6) and latrunculin A-treated samples ( $n=5$; one-way ANOVA with post hoc Scheffé's tests, $p<0.05)$. ${ }^{*} p<0.05,{ }^{* *} p<0.01$. Error bars indicate \pm SEM. 
of F-actin by latrunculin A prolonged the fast-recovery component after STD (Fig. 6C) and impaired fidelity of high-frequency transmission (Fig. 7), indicating that the drug was accessible to the presynaptic target. Nevertheless, latrunculin A treatment had no effect on SV endocytosis at the calyx of Held at PT (Fig. 4D) as well as at RT (Eguchi et al., 2017). More extensive F-actin disassembly might be necessary for blocking SV endocytosis.

Pharmacological depolymerization of F-actin is reported to block ultrafast endocytosis of the subsecond time course (Watanabe et al., 2013), which operates preferentially at PT (Watanabe et al., 2014; Delvendahl et al., 2016). Ultrafast endocytosis takes tens of seconds to reform SVs from endosomes to be reused (Soykan et al., 2017; Watanabe et al., 2018), but it may also contribute to release site clearance, like clathrin-mediated endocytosis (Hosoi et al., 2009; Lee et al., 2012). However, capacitance measurements at the calyx of Held at PT indicated that latrunculin A treatment had no appreciable effect on SV exocytosisendocytosis (Fig. 4D), suggesting that ultrafast endocytosis unlikely operates significantly at the calyx of Held.

High-precision, high-frequency neurotransmission plays pivotal roles in various brain functions, such as sensory processing, cognition, memory formation (Sabatini and Regehr, 1999; Buzsáki and Draguhn, 2004; Uhlhaas and Singer, 2010), and motor control functions (Sugihara et al., 1993). Marked prolongation of recovery from STD and impairments in the fidelity of highfrequency neurotransmission after MT depolymerization (Fig. 6) suggest that presynaptic MTs normally accelerate SV recycling, thereby contributing to the maintenance of integrative synaptic functions.

\section{References}

Arnette C, Frye K, Kaverina I (2016) Microtubule and actin interplay drive intracellular c-Src trafficking. PLoS One 11:e0148996.

Bird MM (1989) Microtubules and their relationships with other cytoskeletal components at cholinergic tectal synapses in culture. J Anat 166:1-6.

Bodaleo FJ, Gonzalez-Billault C (2016) The presynaptic microtubule cytoskeleton in physiological and pathological conditions: lessons from Drosophila fragile X syndrome and hereditary spastic paraplegias. Front Mol Neurosci 9:60.

Buzsáki G, Draguhn A (2004) Neuronal oscillations in cortical networks. Science 304:1926-1929.

Cole JC, Villa BRS, Wilkinson RS (2000) Disruption of actin impedes transmitter release in snake motor terminals. J Physiol 525:579-586.

Darcy KJ, Staras K, Collinson LM, Goda Y (2006) Costitutive sharing of recycling synaptic vesicles between presynaptic boutons. Nat Neurosci 9:315-321.

Delvendahl I, Vyleta NP, von Gersdorff H, Hallermann S (2016) Fast, temperature sensitive and clathrin-independent endocytosis at central synapses. Neuron 90:492-498.

Eguchi K, Taoufiq Z, Thorn-Seshold O, Trauner D, Hasegawa M, Takahashi $\mathrm{T}$ (2017) Wild-type monomeric $\alpha$-synuclein can impair vesicle endocytosis and synaptic fidelity via tubulin polymerization at the calyx of Held. J Neurosci 37:6043-6052.

Etienne-Manneville S (2004) Actin and microtubules in cell motility: which one is in control? Traffic 5:470-477.

Gray EG (1978) Synaptic vesicles and microtubules in frog motor endplates. Proc R Soc Lond B 203:219-227.

Gray EG (1983) Neurotransmitter release mechanisms and microtubules. Proc R Soc Lond B 218:253-258.

Guillaud L, Dimitrov D, Takahashi T (2017) Presynaptic morphology and vesicular composition determine vesicle dynamics in mouse central synapses. eLife 6:e24845.

Hirokawa N, Sobue K, Kanda K, Harada A, Yorifuji H (1989) The cytoskeletal architecture of the presynaptic terminal and molecular structure of synapsin 1. J Cell Biol 108:111-126.

Hirokawa N, Niwa S, Tanaka Y (2010) Molecular motors in neurons: transport mechanisms and roles in brain function, development, and disease. Neuron 68:610-638.
Hori T, Takahashi T (2012) Kinetics of synaptic vesicle refilling with neurotransmitter glutamate. Neuron 76:511-517.

Hosoi N, Holt M, Sakaba T (2009) Calcium dependence of exo-and endocytic coupling at a glutamatergic synapse. Neuron 63:216-229.

Hua Y, Woehler A, Kahms M, Haucke V, Neher E, Klingauf J (2013) Blocking endocytosis enhances short-term synaptic depression under conditions of normal availability of vesicles. Neuron 80:343-349.

Hummel T, Krukkert K, Roos J, Davis G, Klämbt C (2000) Drosophila Futsch/22C10 is a MAP1B-like protein required for dendritic and axonal development. Neuron 26:357-370.

Lee JS, Ho WK, Lee SH (2012) Actin-dependent rapid recruitment of reluctant synaptic vesicles into fast-releasing vesicle pool. Proc Natl Acad Sci U S A 109:E765-E774.

Lepicard S, Franco B, de Bock F, Parmentier ML (2014) A presynaptic role of microtubule-associated protein 1/Futsch in Drosophila: Regulation of active zone number and neurotransmitter release. J Neurosci 34:6759_ 6771.

Lipstein N, Sakaba T, Cooper BH, Lin KH, Strenzke N, Ashery U, Rhee JS, Taschenberger H, Neher E, Brose N (2013) Dynamic control of synaptic vesicle replenishment and short-term plasticity by $\mathrm{Ca}^{2+}$-calmodulinmunc 13-1 signaling. Neuron 79:82-96.

Lukinavičius G, Reymond L, D’Este E, Masharina A, Göttfert F, Ta H, Güther A, Fournier M, Rizzo S, Waldmann H, Blaukopf C, Sommer C, Gerlich DW, Arndt HD, Hell SW, Johnsson K (2014) Fluorogenic probes for live-cell imaging of the cytoskeleton. Nat Methods 11:731-733.

Mahfooz K, Singh M, Renden R, Wesseling JF (2016) A well-defined readily releasable pool with fixed capacity for storing vesicles at calyx of Held. PLoS Comput Biol 12:e1004855.

Malorni W, Quaranta MG, Straface E, Falzano L, Fabbri A, Viora M, Fiorentini C (2003) The rac-activating toxin cytotoxic necrotizing factor 1 oversees NK cell-mediated activity by regulating the actin/microtubule interplay. J Immunol 171:4195-4202.

Melkov A, Abdu U (2018) Regulation of long-distance transport of mitochondria along microtubules. Cell Mol Life Sci 75:163-176.

Miki T, Malagon G, Pulido C, Llano I, Neher E, Marty A (2016) Actin-and myosin-dependent vesicle loading of presynaptic docking sites prior to exocytosis. Neuron 91:808-823.

Morales M, Colicos MA, Goda Y (2000) Actin-dependent regulation of neurotransmitter release at central synapses. Neuron 27:539-550.

Pathak D, Shields LY, Mendelsohn BA, Haddad D, Lin W, Gerencser AA, Kim H, Brand MD, Edwards RH, Nakamura K (2015) The role of mitochondrially derived ATP in synaptic vesicle recycling. J Biol Chem 290:2232522336.

Perkins GA, Tjong J, Brown JM, Poquiz PH, Scott RT, Kolson DR, Ellisman MH, Spirou GA (2010) The micro-architecture of mitochondria at active zones: electron tomography reveals novel anchoring scaffolds and cristae structured for high-rate metabolism. J Neurosci 30:10151026.

Pusch M, Neher E (1988) Rates of diffusional exchange between small cells and a measuring patch pipette. Pflugers Arch 411:204-211.

Sabatini BL, Regehr WG (1999) Timing of synaptic transmission. Annu Rev Physiol 61:521-542.

Saitoh N, Hori T, Takahashi T (2001) Activation of the epsilon isoform of protein kinase $\mathrm{C}$ in the mammalian nerve terminal. Proc Natl Acad Sci U S A 98:14017-14021.

Sakaba T, Neher E (2001) Calmodulin mediates rapid recruitment of fastreleasing synaptic vesicles at a calyx-type synapse. Neuron 32:11191131.

Sakaba T, Neher E (2003) Involvement of actin polymerization in vesicle recruitment at the calyx of Held synapse. J Neurosci 23:837-846.

Sakaba T, Kononenko NL, Bacetic J, Pechstein A, Schmoranzer J, Yao L, Barth H, Shupliakov O, Kobler O, Aktories K, Haucke V (2013) Fast neurotransmitter release regulated by the endocytic scaffold intersectin. Proc Natl Acad Sci U S A 110:8266-8271.

Schneggenburger R, Meyer AC, Neher E (1999) Released fraction and total size of a pool of immediately available transmitter quanta at a calyx synapse. Neuron 23:399-409.

Smith DS, Järlfors U, Beránek R (1970) The organization of synaptic axoplasm in the lamprey (Petromyzon marinus) central nervous system. J Cell Biol 46:199-219.

Soykan T, Kaempf N, Sakaba T, Vollweiter D, Goerdeler F, Puchkov D, Kononenko NL, Haucke V (2017) Synaptic vesicle endocytosis occurs 
on multiple timescales and is mediated by formin-dependent actin assembly. Neuron 93:854-866.e4.

Sugihara I, Lang EJ, Llinás R (1993) Uniform olivocerebellar conduction time underlies Purkinje cell complex spike synchronicity in the rat cerebellum. J Physiol 470:243-271.

Sun JY, Wu XS, Wu LG (2002) Single and multiple vesicle fusion induce different rates of endocytosis at a central synapse. Nature 417:555559.

Takahashi T, Hori T, Kajikawa Y, Tsujimoto T (2000) The role of GTPbinding protein activity in fast central synaptic transmission. Science 289:460-463.

Uhlhaas PJ, Singer W (2010) Abnormal neural oscillations and synchrony in schizophrenia. Nat Rev Neurosci 11:100-113.

Wang LY, Kaczmarek LK (1998) High-frequency firing helps replenish the readily releasable pool of synaptic vesicles. Nature 394:384-388.

Watanabe S, Rost BR, Camacho-Pérez M, Davis MW, Söhl-Kielczynski B,
Rosenmund C, Jorgensen EM (2013) Ultrafast endocytosis at mouse hippocampal synapses. Nature 504:242-247.

Watanabe S, Trimbuch T, Camacho-Pérez M, Rost BR, Brokowski B, SöhlKielczynski, Felies A, Davis MW, B, Rosenmund C, Jorgensen EM (2014) Clathrin regenerates synaptic vesicles from endosomes. Nature 515:228 233.

Watanabe S, Mamer LE, Raychaudhuri S, Luvsanjav D, Eisen J, Trimbuch T, Söhl-Kielczynski B, Fenske P, Milosevic I, Rosenmund C, Jorgensen EM (2018) Synaptojanin and endophilin mediate neck formation during ultrafast endocytosis. Neuron 98:1184-1197.e6.

Wu XS, Lee SH, Sheng J, Zhang Z, Zhao WD, Wang D, Jin Y, Charnay P, Ervasti JM, Wu LG (2016) Actin is crucial for all kinetically distinguishable forms of endocytosis at synapses Neuron 92:1020-1035.

Yamashita T, Hige T, Takahashi T (2005) Vesicle endocytosis requires dynamin dependent GTP hydrolysis at a fast CNS synapse. Science 307:124127. 